\title{
Analysis of the Block-Grid Method for the Solution of Laplace's Equation on Polygons with a Slit
}

\author{
S. Cival Buranay \\ Department of Mathematics, Eastern Mediterranean University, Gazimagusa, Cyprus, 095 Mersin 10, Turkey \\ Correspondence should be addressed to S. Cival Buranay; suzan.buranay@emu.edu.tr
}

Received 22 April 2013; Accepted 30 April 2013

Academic Editor: Allaberen Ashyralyev

Copyright (C) 2013 S. Cival Buranay. This is an open access article distributed under the Creative Commons Attribution License, which permits unrestricted use, distribution, and reproduction in any medium, provided the original work is properly cited.

\begin{abstract}
The error estimates obtained for solving Laplace's boundary value problem on polygons by the block-grid method contain constants that are difficult to calculate accurately. Therefore, the experimental analysis of the method could be essential. The real characteristics of the block-grid method for solving Laplace's equation on polygons with a slit are analysed by experimental investigations. The numerical results obtained show that the order of convergence of the approximate solution is the same as in the case of a smooth solution. To illustrate the singular behaviour around the singular point, the shape of the highly accurate approximate solution and the figures of its partial derivatives up to second order are given in the "singular" part of the domain. Finally a highly accurate formula is given to calculate the stress intensity factor, which is an important quantity in fracture mechanics.
\end{abstract}

\section{Introduction}

In the past few decades, in order to improve the accuracy and resolve the convergence difficulties that appear in the neighbourhood of singular points, many different methods have been proposed for the numerical solution of plane elliptic boundary value problems with singularities. Among many approaches, a special emphasis has been placed on the construction of combined methods, in which differential properties of the solution in different parts of the domain are used (see [1]).

In [2-6] a new combined difference-analytical method called the block-grid method (BGM) is given for solving the Laplace equation on polygons, when the boundary functions on the sides causing the singular vertices are given as algebraic polynomials of the arclength. This method is a combination of the exponentially convergent block method (see $[7,8]$ ) in the "singular" part, and the finite difference method, which has a simple structure on the "nonsingular" part of the polygon. A $k$ th order gluing operator $S^{k}$ is constructed for gluing together the grids and the blocks. The uniform estimate of the error of the BGM is of order $O\left(h^{k}\right)$ ( $h$ is the mesh step) when the given boundary function on the boundary of the "nonsingular" part might be from the Hölder classes $C^{k, \lambda}, 0<\lambda<1$ (see [2-4] for $k=6$, [6] for $k=4$, and [5] for $k=2$ ). For the errors of $p$ order derivatives $(p=1,2, \ldots)$ the estimation $O\left(h^{k} / r_{j}^{p-1 / \alpha_{j}}\right)$ is obtained in a finite neighborhood of the vertices, where $r_{j}$ is the distance from the current point to the vertex in question, and $\alpha_{j} \pi$ is the value of the interior angle at the considered vertex. Moreover, BGM can give a simple and highly accurate formula for the stress intensity factor which is an important quantity from an engineering standpoint.

The experimental investigation of the block-grid method is important and numerical results could be interesting to support the theoretical results in [2-6]. The objective of this paper is to analyze the real characteristics of the BGM for solving the Laplace equation on polygons with a slit. For this purpose a slit problem on a square domain whose exact solution is known is considered. The computational algorithm by the BGM with 5-point and 9-point schemes is given and implemented. The obtained numerical results justify the theoretical results given in [2-5]. Moreover, for the approximate solution $U_{h}^{6}$ (by 9-point scheme with $S^{6}$ ) and the error function the graphs are given to demonstrate the high accuracy of the block-grid method. The shapes of the partial derivatives $\partial U_{h}^{6} / \partial x, \partial U_{h}^{6} / \partial y, \partial^{2} U_{h}^{6} / \partial x^{2}, \partial^{2} U_{h}^{6} / \partial y^{2}$, $\partial^{2} U_{h}^{6} / \partial x \partial y$ are given to illustrate the singular behavior in the 
"singular" part of the domain. Furthermore, a simple and highly accurate formula is given to calculate the stress intensity factor.

The experimental analyses of the different methods on slit problems were given in many papers (see $[9,10])$.

\section{The Slit Problem and the Integral Representation of the Solution}

Let $G$ be an open domain in the plane $x O y$, that is obtained from the unit square $G=\{(x, y):|x|<1,|y|<1\}$ by making a cut $O A$ along the positive semiaxis $O x$ from the center (see Figure 1). Let $\gamma_{j}, j=1(1) 7$, be its sides, including the ends, enumerated counterclockwise, $\gamma=\gamma_{1} \cup \cdots \cup \gamma_{7},\left(\gamma_{0}=\gamma_{7}\right)$, be the boundary of $G, 2 \pi$ is the interior angle formed by the sides $\gamma_{1}$ and $\gamma_{0}$. Denote by $O=\gamma_{0} \cap \gamma_{1}$ the vertex of this angle and let $r, \theta$ be a polar system of coordinates with a pole in $O$, where the angle $\theta$ is taken counterclockwise from the side $\gamma_{1}$.

We consider the boundary value problem

$$
\begin{gathered}
\Delta u=0 \quad \text { on } G, \\
u=\varphi_{j} \quad \text { on } \gamma_{j}, j=1,2, \ldots, 7,
\end{gathered}
$$

where $\Delta \equiv \partial^{2} / \partial x^{2}+\partial^{2} / \partial y^{2}$ and $\varphi_{j}$ is the value of the function $v(r, \theta)=\sqrt{2} r^{1 / 2} \sin (1 / 2) \theta$ on $\gamma_{j}$.

In the neighborhood of $O$, we construct two fixed blocksectors $T^{i}=T\left(r_{i}\right) \subset G, i=1,2$, where $0<r_{2}<r_{1}<1$, $T(\rho)=\{(r, \theta): 0<r<\rho, 0<\theta<2 \pi\} \subset G$.

Let

$$
R_{1}(r, \theta, \eta)=\frac{1}{2} \sum_{k=0}^{1}(-1)^{k} R\left(\left(\frac{r}{r_{2}}\right)^{1 / 2}, \frac{\theta}{2},(-1)^{k} \frac{\eta}{2}\right),
$$

where

$$
R(r, \theta, \eta)=\frac{1-r^{2}}{2 \pi\left(1-2 r \cos (\theta-\eta)+r^{2}\right)}
$$

is the kernel of the Poisson integral for a unit circle.

Lemma 1. The solution $u$ of the boundary value problem (1), (2) can be represented on $\bar{T}^{2} \backslash V$, in the form

$$
u(r, \theta)=\int_{0}^{2 \pi} R_{1}(r, \theta, \eta) u\left(r_{2}, \eta\right) d \eta
$$

where $V$ is the curvilinear part of the boundary of $T^{2}$.

Proof. The proof follows from Theorem 3.1 in [8] by taking into account that $\varphi_{0}=\varphi_{1}=0$.

\section{The Block-Grid Method for the Slit Problem}

The realization of the BGM for the solution of the problem (1), (2) is as follows. Let $T^{2}=T(0.93)$ and $t$ be a polygonal line $a b c d e$ which lies on $T^{2}$ with a positive distance from the vertex $O$ and from the curvilinear boundary $V=\{(r, \theta)$ :

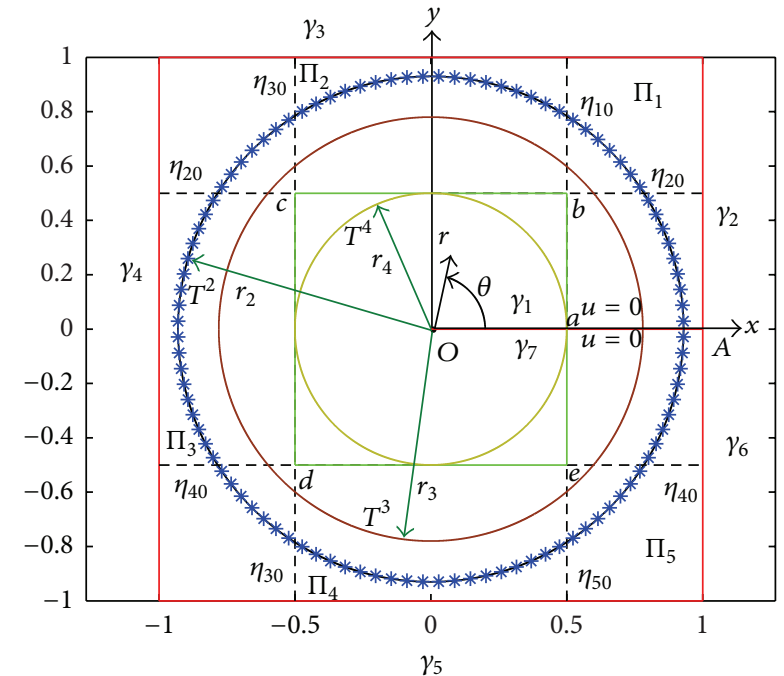

FIgURE 1: Covering the square domain with a slit by overlapping rectangles and sector.

$r=0.93,0<\theta<2 \pi\}$ of $T^{2}$. The set of points $T^{2}$ from $O$ up to $t$ is denoted by $G^{\text {S }}$ which is called the "singular" part of $G$ and the set $G^{\mathrm{NS}}=G \backslash G^{\mathrm{S}}$ is the "nonsingular" part of $G$. In addition to the sector $T^{2}$ in the neighborhood of the vertex $O$ of the polygon $G$ we construct two more sectors $T^{3}=T(0.85)$ and $T^{4}=T(\sqrt{5})$. Let $G_{T}=G \backslash\left(T^{4}\right)$ and $\Pi_{l} \subset G^{\mathrm{NS}} \subset G_{T}$, $l=1(1) 5$, be fixed open rectangles (see Figure 1). Then the domain $G$ can be represented as $G=\left(\cup_{l=1}^{5} \Pi_{l}\right) \cup\left(T^{3}\right)$. Let $\eta_{l}$ be the boundary of the rectangle $\Pi_{l}$ and $t_{l}=\eta_{l} \cap t$. We define a square grid on $\Pi_{l}, l=1(1) 5$, with step $h$ such that the boundary $\eta_{l}$ lies entirely on the grid lines. $\Pi_{l}^{h}$ denotes the set of grid nodes on $\Pi_{l}, \eta_{l}^{h}$ denotes the set of nodes on $\eta_{l}$ and $\bar{\Pi}_{l}^{h}=\Pi_{l}^{h} \cup \eta_{l}^{h}$. We refer to the set of nodes on the closure of $\eta_{l} \cap G_{T}$ as $\eta_{l 0}^{h}$, the set of nodes on $t_{l}$ as $t_{l}^{h}$ and the set of remaining nodes on $\eta_{l}$ as $\eta_{l 1}^{h}$. We also introduce the natural number $n \geq 4$, and $\theta^{q}=(q-1 / 2) 2 \pi / n, 1 \leq q \leq n$. On the arc $V$, we choose the points $\left(0.93, \theta^{q}\right), 1 \leq q \leq n$, denote the set of these points by $V^{n}$ and let $\bar{G}_{T}^{h, n}=V^{n} \cup\left(\cup_{l=1}^{5} \bar{\Pi}_{l}^{h}\right)$.

Let $\varphi=\left\{\varphi_{j}\right\}_{j=1}^{7}$, where $\varphi_{j}$ is the given function in (2). We introduce a gluing operator $S^{k}, k=2,6$ ([5] for $k=2$ and [24] for $k=6)$ at the points of the set $V^{n}$. We denote by $u_{h}^{k}\left(U_{h}^{k}\right)$ the approximate solution of the problem (1), (2) obtained by the 5-point scheme with $S^{2}$ for $k=2$, and by the 9-point scheme with $S^{6}$ for $k=6$, on the "singular" ("nonsingular") part of $G$. The operator $S^{2}$ is defined at each point $P \in V^{n}$ in the following way: we consider the set of all rectangles $\left\{\Pi_{l}\right\}$ in the intersections of which the point $P$ lies, and we choose one of these rectangles $\Pi_{l(P)}$ part of whose boundary situated in $G^{T}$ is furthest away from $P$. The value $S^{2}\left(u_{h}^{2}, \varphi\right)$ at the point $P \in V^{n}$ is computed according to the values of the function at the four vertices $P_{\kappa}, \kappa=1(1) 4$, of the closure of the cell, containing the point $P$ of the grid constructed on $\bar{\Pi}_{l(P)}$ by multilinear interpolation. 
The value of $S^{6}\left(u_{h}^{6}, \varphi\right)$ at the point $P \in V^{n}$ is expressed linearly in terms of the values of $u_{h}^{6}$ at the points $P_{\kappa}, \kappa=$ 1(1)31, of the grid constructed on $\Pi_{l(P)} \ni P$ some part of whose boundary located in $G$ is the maximum distance away from $P$, and in terms of the boundary values of $\varphi^{(\tau)}, \tau=$ $0,1, \ldots, 5$ at a fixed number of points. Moreover, $S^{k}\left(u_{h}^{k}, 0\right)$ has the representation

$$
S^{k}\left(u_{h}^{k}, 0\right)= \begin{cases}\sum_{1 \leq \kappa \leq 31} \xi_{\kappa} u_{h, \kappa}^{k}, & \text { for } k=6, \\ \sum_{1 \leq \kappa \leq 4} \lambda_{\kappa} u_{h, \kappa}^{k}, & \text { for } k=2,\end{cases}
$$

where $u_{h, \kappa}^{k}=u_{h}^{k}\left(P_{\kappa}\right)$,

$$
\xi_{\kappa} \geq 0, \quad \sum_{1 \leq \kappa \leq 31} \xi_{\kappa}=1, \quad \lambda_{\kappa} \geq 0, \quad \sum_{1 \leq \kappa \leq 4} \lambda_{\kappa}=1,
$$

and for the exact solution $u$ of the problem (1), (2), we have

$$
\begin{aligned}
& u-S^{6}(u, \varphi)=O\left(h^{6}\right), \\
& u-S^{2}(u, \varphi)=O\left(h^{2}\right) .
\end{aligned}
$$

Remark 2. Let $V_{I}^{n} \subset V^{n}$ be the set of such points $P \in V^{n}$, for which all points $P_{\kappa}$ in the expression (6) are in $\cup_{l=1}^{5} \bar{\Pi}_{l}^{h}$. If some of the points $P_{\kappa}$ in (6) emerge through the side $\gamma_{j}$ when $u=\varphi_{j}, 1 \leq j \leq 7$, we denote the set of such points $P$ by $V_{D}^{n}$. Then, according to the construction of $S^{6}$ in [4] the expression $S^{6}\left(u_{h}^{6}, \varphi\right)$ at each point $P \in V^{n}=V_{I}^{n} \cup V_{D}^{n}$ can be expressed as follows:

$$
\begin{aligned}
& S^{6}\left(u_{h}^{6}, \varphi\right) \\
& = \begin{cases}S^{6} u_{h}^{6}, & P \in V_{I}^{n}, \\
S^{6}\left(u_{h}^{6}-\sum_{\tau=0}^{5} a_{\tau} \operatorname{Re} z^{\tau}\right) & \\
+\left(\sum_{\tau=0}^{5} a_{\tau} \operatorname{Re} z^{\tau}\right)_{P}, & P \in V_{D}^{n},\end{cases}
\end{aligned}
$$

where

$$
a_{\tau}=\left.\frac{1}{\tau !} \frac{d^{\tau} \varphi_{j}(s)}{d s^{\tau}}\right|_{s=s_{P}}, \quad \tau=0,1, \ldots, 5 .
$$

$s_{P}$ corresponds to such point $Q \in \gamma_{j}$ for which the line $P Q$ is perpendicular to $\gamma_{j}$.

Consider for each $k=2,6$ the following system of linear algebraic equations:

$$
\begin{gathered}
u_{h}^{k}=B_{k} u_{h}^{k} \quad \text { on } \Pi_{l}^{h}, \\
u_{h}^{k}=\sqrt{2} r^{1 / 2} \sin \frac{1}{2} \theta \quad \text { on } \eta_{l 1}^{h} \cap \gamma_{j}, \\
u_{h}^{k}(r, \theta)=\frac{2 \pi}{n} \sum_{q=1}^{n} u_{h}^{k}\left(0.93, \theta^{q}\right) \\
\times R_{1}\left(r, \theta, \theta^{q}\right) \quad \text { on }(r, \theta) \in t_{l}^{h}, \\
u_{h}^{k}=S^{k} u_{h}^{k} \quad \text { on } V^{n}, 1 \leq l \leq 5,1 \leq j \leq 7,
\end{gathered}
$$

where

$$
\begin{aligned}
& B_{6} u(x, y) \equiv( u(x+h, y)+u(x, y+h)+u(x-h, y) \\
&+u(x, y-h)) / 5 \\
&+(u(x+h, y+h) \\
&+u(x-h, y+h)+u(x-h, y-h) \\
&+u(x+h, y-h)) / 20, \\
& B_{2} u(x, y) \equiv(u(x+h, y)+u(x, y+h) \\
&+ \\
&\quad u(x-h, y)+u(x, y-h)) / 4 .
\end{aligned}
$$

Theorem 3. There is a natural number $n_{0}$ such that for all $n \geq$ $n_{0}$, and for each $k=2,6$, the system (11)-(14) has a unique solution.

Proof. The proof follows when $k=2$ from [5], and when $k=$ 6 from $[3,4]$.

We consider the sector $T^{*}=T(0.89)$, and let $u_{h}^{k}\left(0.93, \theta^{q}\right)$, $1 \leq q \leq n$, be the values of the solution of the system (11)-(14) on $V^{n}$ (at the quadrature nodes). The function

$$
U_{h}^{k}(r, \theta)=\frac{2 \pi}{n} \sum_{q=1}^{n} R_{1}\left(r, \theta, \theta^{q}\right) u_{h}^{k}\left(0.93, \theta^{q}\right),
$$

defined on $T^{*}$ is called an approximate solution of the problem (1), (2) on the closed block $\bar{T}^{3}$.

Everywhere below we will denote constants which are independent of $h$ and of the cofactors on their right by $c, c_{0}, c_{1}$ for simplicity.

Theorem 4. There exists a natural number $n_{0}$ such that for

$$
n \geq \max \left\{n_{0},\left[\ln ^{1+\varkappa} h^{-1}\right]+1\right\},
$$

where $u>0$ is a fixed number, the following inequalities are valid:

$$
\begin{gathered}
\max _{\bar{G}_{T}^{h, n}}\left|u_{h}^{k}-u\right| \leq c h^{k}, \\
\left|\left(U_{h}^{k}(r, \theta)-u(r, \theta)\right)\right| \leq c_{0} r^{1 / 2} h^{k} \quad \text { on } \bar{T}^{3}, \\
\left|\frac{\partial^{p}}{\partial x^{p-q} \partial y^{q}}\left(U_{h}^{k}(r, \theta)-u(r, \theta)\right)\right| \\
\leq c_{1} h^{k} / r^{p-(1 / 2)} \text { on } \bar{T}^{3} \backslash O,
\end{gathered}
$$

for all $p=1,2, \ldots$ Everywhere $0 \leq q \leq p, u$ is a solution of the problem (1), (2).

Proof. The proof is carried out analogically to the proof of Theorems 1 and 2 in [3]. 


\section{Computational Algorithm}

Let $\Pi=\left\{(x, y): a_{1}<x<a_{2}, b_{1}<y<b_{2}\right\}$, where $a_{2}-$ $a_{1}=2^{p} h_{0}, b_{2}-b_{1}=2^{q} h_{0}, h_{0}>0$ is a fixed number, and $p$ and $q$ are integers. We introduce a square grid with the lines $x=a_{1}+i h, y=b_{1}+j h, h=h_{0} 2^{-m}, m \geq 0$ is an integer, $i=0,1, \ldots, 2^{p+m}, j=0,1, \ldots, 2^{q+m}$. Let $\Pi_{h}=\{(x, y): x=$ $\left.x_{i}=a_{1}+i h, 0<i<2^{p+m}, y=y_{j}=b_{1}+j h, 0<j<2^{q+m}\right\}$ and $\Gamma_{h}=\Gamma_{1 h} \cup \Gamma_{2 h} \cup \Gamma_{3 h} \cup \Gamma_{4 h}$ be a set of nodes on $\Gamma$ (the boundary of $\Pi)$ where

$$
\begin{aligned}
& \Gamma_{1 h}=\left\{(x, y): x=a_{1}+i h, 1 \leq i \leq 2^{p+m}, y=b_{1}\right\}, \\
& \Gamma_{2 h}=\left\{(x, y): x=a_{2}, y=b_{1}+j h, 1 \leq j \leq 2^{q+m}\right\}, \\
& \Gamma_{3 h}=\left\{(x, y): x=a_{1}+i h, 1 \leq i \leq 2^{p+m}, y=b_{2}\right\}, \\
& \Gamma_{4 h}=\left\{(x, y): x=a_{1}, y=b_{1}+j h, 1 \leq j \leq 2^{q+m}\right\} .
\end{aligned}
$$

We consider for each $k=2,6$ the finite difference problem

$$
\begin{gathered}
u_{h}^{k}=B_{k} u_{h}^{k} \quad \text { on } \Pi_{h}, \\
u_{h}^{k}=\varphi_{j h} \quad \text { on } \Gamma_{j h}, j=1,2,3,4,
\end{gathered}
$$

where $\varphi_{j h}$ is a given function on $\Gamma_{j h}$ that vanishes at the end points.

The solution of the problem (25) can be found using the superposition principle $u_{h}^{k}=u_{1 h}^{k}+u_{2 h}^{k}+u_{3 h}^{k}+u_{4 h}^{k}$ as the sum of solution of four problems of the type

$$
\begin{gathered}
u_{j h}^{k}=B_{k} u_{h} \quad \text { on } \Pi_{h}, \\
u_{j h}^{k}= \begin{cases}\varphi_{j h} & \text { on } \Gamma_{j h}, \\
0 & \text { on } \Gamma_{h} \backslash \Gamma_{j h}, \quad j=1,2,3,4 .\end{cases}
\end{gathered}
$$

The solution of the problem (26), when $j=1$ has the representation

$$
\begin{aligned}
& u_{1 h}^{k}(x, y) \\
& \quad=\sum_{n=1}^{2^{p+m}-1} d_{n} \frac{\sinh \left(\beta_{n}^{k}\left(1-y /\left(b_{2}-b_{1}\right)\right)\right)}{\sinh \beta_{n}^{k}} \sin \frac{n \pi x}{a_{2}-a_{1}}, \\
& d_{n}=2^{1-p-m} \sum_{r=1}^{2^{p+m}-1} \varphi_{1 h}\left(a_{1}+r h\right) \sin \frac{n \pi\left(a_{1}+r h\right)}{a_{2}-a_{1}},
\end{aligned}
$$

where

$$
\beta_{n}^{2}=\frac{2\left(b_{2}-b_{1}\right)}{h} \sinh ^{-1}\left(\sin \frac{n \pi h}{2\left(a_{2}-a_{1}\right)}\right),
$$

for the 5-point approximation [11],

$$
\begin{aligned}
\beta_{n}^{6}= & \frac{2\left(b_{2}-b_{1}\right)}{h} \\
& \times \sinh ^{-1}\left(\frac{\sin n \pi h / 2\left(a_{2}-a_{1}\right)}{\sqrt{1-2 \sin ^{2}\left(n \pi h / 2\left(a_{2}-a_{1}\right)\right) / 3}}\right),
\end{aligned}
$$

for the 9-point approximation [12].
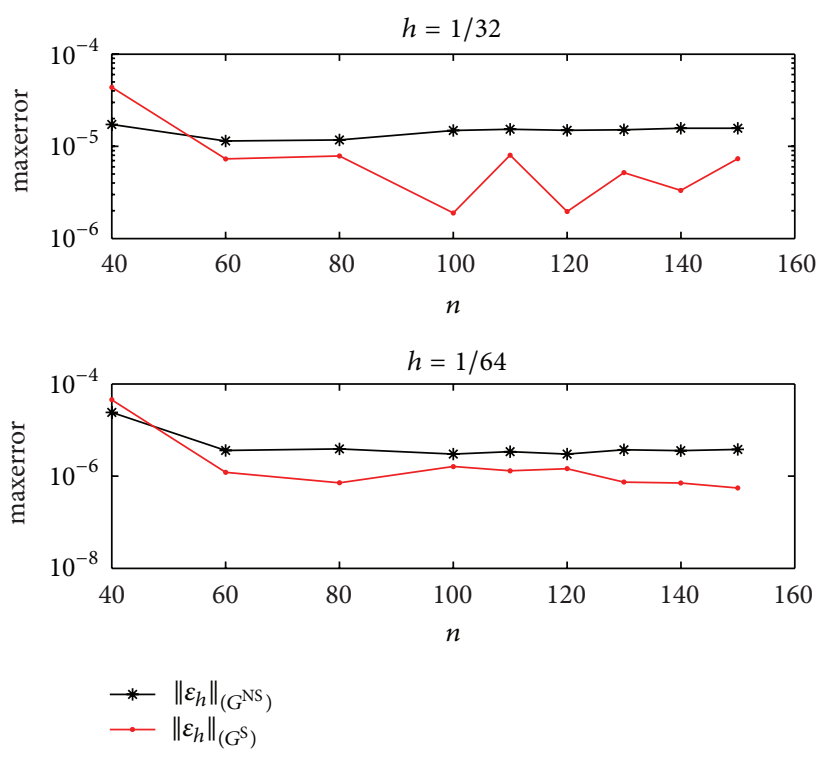

FIGURE 2: The errors with respect to number of quadrature nodes $n$, in the "singular" part and in the "nonsingular" part by the BGM when 5-point scheme is used with $S^{2}$ for $h^{-1}=32,64$.
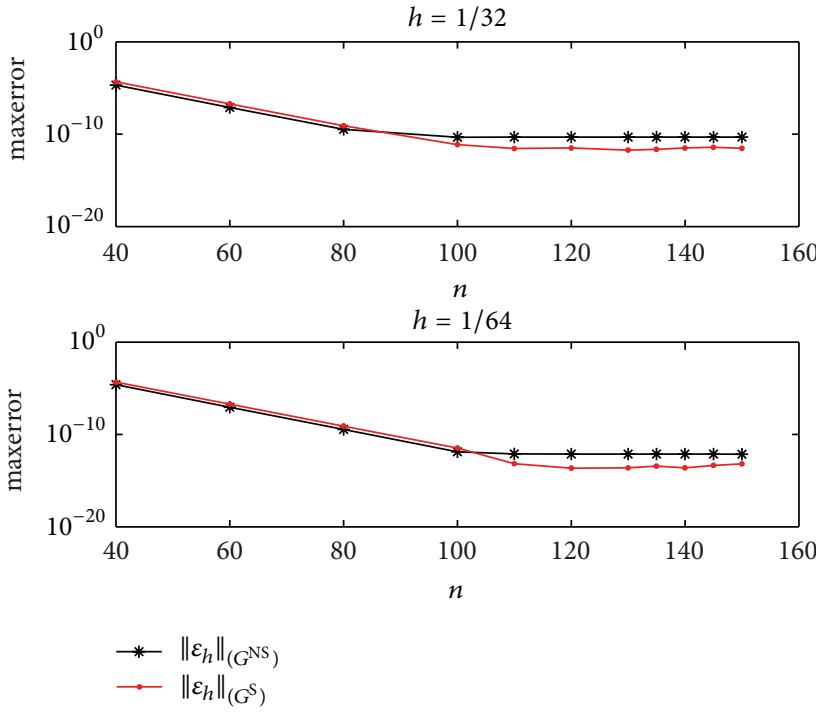

FIGURE 3: The errors with respect to number of quadrature nodes $n$, in the "singular" part and in the "nonsingular" part by the BGM when 9-point scheme is used with $S^{6}$ for $h^{-1}=32,64$.

The Discrete Fast Fourier Transform is used for the realization of the finite sums in (27). The solution of the problem (26), for $j=2,3,4$ can be represented analogously.

Now we describe the algorithm of implementing the BGM for the slit problem.

Step 1. Suppose that we have zero approximation $u_{h}^{k(0)}$ to the exact solution $u_{h}^{k}$ of (11)-(14). 


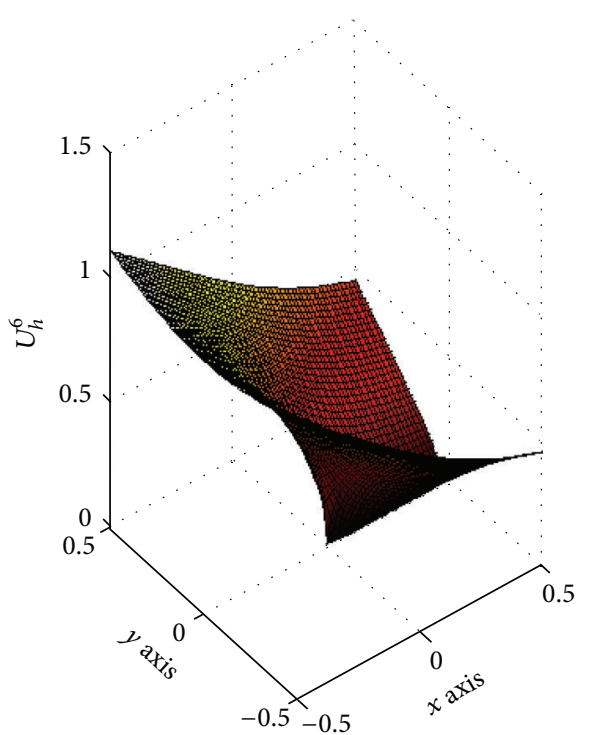

(a)

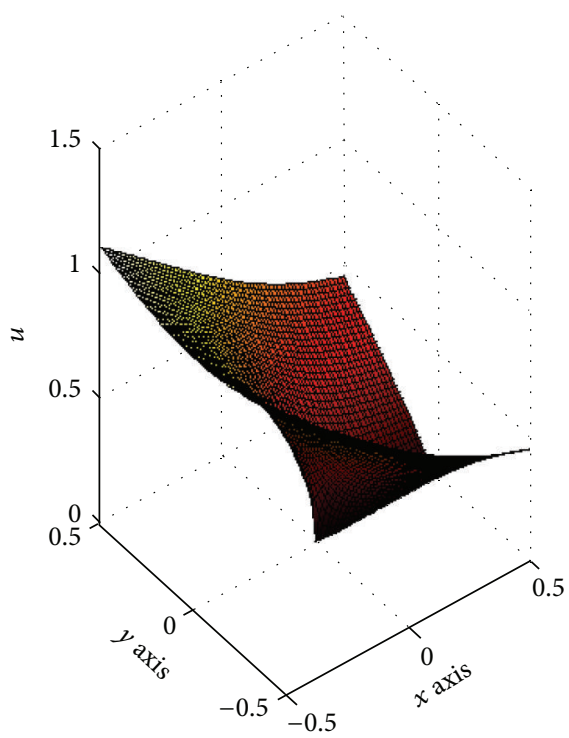

(b)

FIGURE 4: The highly accurate approximate solution $U_{h}^{6(M)}$ and the exact solution $u$ in the "singular" part for $h^{-1}=64, n=140$.

TABLE 1: The errors by BGM when 5-point scheme is used with $S^{2}$ interpolation.

\begin{tabular}{lcccc}
\hline$\left(h^{-1}, n\right)$ & $\left\|\varepsilon_{h}\right\|_{\left(G^{\mathrm{NS}}\right)}$ & $\left\|\varepsilon_{h}\right\|_{\left(G^{\mathrm{S}}\right)}$ & $\left\|\varepsilon_{h}^{(1)}\right\|_{\left(G^{\mathrm{S}}\right)}$ & $\left\|\varepsilon_{h}^{(2)}\right\|_{\left(G^{\mathrm{S}}\right)}$ \\
\hline$(16,85)$ & $6.138 \times 10^{-5}$ & $2.3127 \times 10^{-5}$ & $6.432 \times 10^{-5}$ & $4.464 \times 10^{-4}$ \\
$(16,100)$ & $5.264 \times 10^{-5}$ & $1.393 \times 10^{-5}$ & $5.126 \times 10^{-5}$ & $3.244 \times 10^{-4}$ \\
$(16,120)$ & $5.599 \times 10^{-5}$ & $1.150 \times 10^{-5}$ & $5.385 \times 10^{-5}$ & $3.430 \times 10^{-4}$ \\
$(32,85)$ & $1.317 \times 10^{-5}$ & $4.676 \times 10^{-6}$ & $1.129 \times 10^{-5}$ & 12 \\
$(32,100)$ & $1.488 \times 10^{-5}$ & $1.889 \times 10^{-6}$ & $2.056 \times 10^{-5}$ & $1.272 \times 10^{-5}$ \\
$(32,120)$ & $1.491 \times 10^{-5}$ & $1.956 \times 10^{-6}$ & $2.053 \times 10^{-5}$ & $1.740 \times 10^{-4}$ \\
$(32,130)$ & $1.508 \times 10^{-5}$ & $5.172 \times 10^{-6}$ & $1.728 \times 10^{-5}$ & $4.659 \times 10^{-5}$ \\
$(32,140)$ & $1.571 \times 10^{-5}$ & $3.319 \times 10^{-6}$ & $2.407 \times 10^{-5}$ & $2.155 \times 10^{-4}$ \\
$(64,130)$ & $3.720 \times 10^{-6}$ & $7.391 \times 10^{-7}$ & $2.306 \times 10^{-6}$ & 13 \\
$(64,140)$ & $3.583 \times 10^{-6}$ & $7.071 \times 10^{-7}$ & $3.852 \times 10^{-6}$ & 13 \\
\hline & & & & $13.941 \times 10^{-5}$ \\
\end{tabular}

Step 2. Finding $u_{h}^{k(1)}$ by the formula (13) on $t_{l}^{h}$ we solve the system (11), (12) on each grid $\bar{\Pi}_{l}^{h}$ by using the representation of finite difference solution described before Step 1.

Step 3. Using (6) we calculate the values $u_{h}^{k(1)}\left(0.93, \theta^{q}\right)$ at the quadrature nodes for each $\theta^{q}=(q-1 / 2) 2 \pi / n, 1 \leq q \leq n$ by the formula (14).

Step 4. Repeating Steps 2 and 3 we have the sequence $u_{h}^{k(1)}, u_{h}^{k(2)}, \ldots$, of Schwarz's iterations defined as follows:

$$
\begin{aligned}
u_{h}^{k(m)}(r, \theta)=\frac{2 \pi}{n} \sum_{q=1}^{n} & R_{1}\left(r, \theta, \theta^{q}\right) \\
& \times(r, \theta) u_{h}^{k(m-1)}\left(0.93, \theta^{q}\right) \text { on } t_{l}^{h}, \\
u_{h}^{k(m)} & =S^{k} u_{h}^{k(m-1)} \text { on } V^{n},
\end{aligned}
$$

$$
\begin{gathered}
u_{h}^{k(m)}=B_{k} u_{h}^{k(m)} \quad \text { on } \Pi_{l}^{h}, \\
u_{h}^{k(m)}=\varphi \quad \text { on } \eta_{l 1}^{h}, k=2,6,1 \leq l \leq 5, m=1,2, \ldots
\end{gathered}
$$

As a stopping criteria of the Schwarz's iterations (30), we use the inequality $\max _{\eta_{l 0}, l=1,2, \ldots, 5}\left|u_{h}^{k(m)}-u_{h}^{k(m-1)}\right| \leq \epsilon$ for the prescribed accuracy of $\epsilon>0$.

Step 5. Let $u_{h}^{k(M)}\left(0.93, \theta^{q}\right), \theta^{q}=(q-1 / 2) 2 \pi / n, 1 \leq q \leq n$, in (14) be the values at the quadrature nodes on $V^{n}$ for the final iteration $m=M$. Using these values we can calculate the value of the solution at any point in the singular part by the explicit formula

$$
U_{h}^{k(M)}(r, \theta)=\frac{2 \pi}{n} \sum_{q=1}^{n} R_{1}\left(r, \theta, \theta^{q}\right) u_{h}^{k(M)}\left(0.93, \theta^{q}\right)
$$


TABLE 2: The errors by BGM when 9-point scheme is used with $S^{6}$ interpolation.

\begin{tabular}{lcccc}
\hline$\left(h^{-1}, n\right)$ & $\left\|\varepsilon_{h}\right\|_{\left(G^{\mathrm{NS}}\right)}$ & $\left\|\varepsilon_{h}\right\|_{\left(G^{\mathrm{S}}\right)}$ & $\left\|\varepsilon_{h}^{(1)}\right\|_{\left(G^{\mathrm{S}}\right)}$ & $\left\|\varepsilon_{h}^{(2)}\right\|_{\left(G^{\mathrm{S}}\right)}$ \\
\hline$(16,100)$ & $2.741 \times 10^{-9}$ & $4.895 \times 10^{-10}$ & $6.778 \times 10^{-10}$ & $2.293 \times 10^{-9}$ \\
$(16,145)$ & $2.789 \times 10^{-9}$ & $3.786 \times 10^{-10}$ & $5.108 \times 10^{-10}$ & $1.859 \times 10^{-9}$ \\
$(32,100)$ & $4.706 \times 10^{-11}$ & $7.158 \times 10^{-12}$ & $2.593 \times 10^{-10}$ & $4.691 \times 10^{-9}$ \\
$(32,125)$ & $4.805 \times 10^{-11}$ & $2.694 \times 10^{-12}$ & $6.623 \times 10^{-12}$ & $4.358 \times 10^{-11}$ \\
$(32,130)$ & $4.838 \times 10^{-11}$ & $1.831 \times 10^{-12}$ & $7.257 \times 10^{-12}$ & $5.186 \times 10^{-11}$ \\
$(32,145)$ & $4.745 \times 10^{-11}$ & $3.903 \times 10^{-12}$ & $8.808 \times 10^{-12}$ & $5.379 \times 10^{-11}$ \\
$(64,125)$ & $7.856 \times 10^{-13}$ & $2.220 \times 10^{-14}$ & $1.296 \times 10^{-12}$ & $3.364 \times 10^{-11}$ \\
$(64,130)$ & $7.545 \times 10^{-13}$ & $4.097 \times 10^{-14}$ & $5.376 \times 10^{-13}$ & 24 \\
$(64,145)$ & $7.503 \times 10^{-13}$ & $4.396 \times 10^{-14}$ & $1.614 \times 10^{-13}$ & $24 \times 10^{-11}$ \\
\hline
\end{tabular}

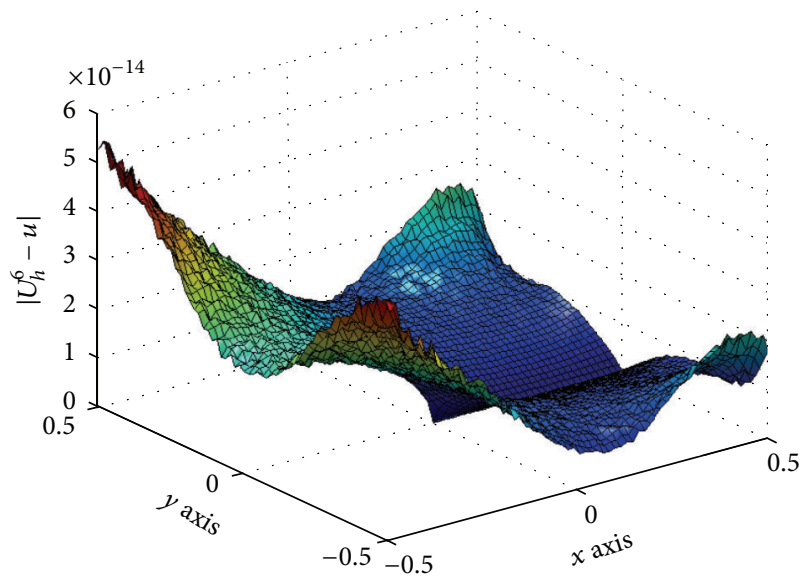

Figure 5: The error $\left|U_{h}^{6(M)}-u\right|$ in the "singular" part for $h^{-1}=64$, $n=140$.

TABLE 3: The order of convergence $R_{G^{\mathrm{NS}}}^{2, \varrho}$, and $R_{G^{S}}^{2, \varrho}$ when $h=2^{-\varrho}$.

\begin{tabular}{lcc}
\hline$\left(2^{-\varrho}, n\right)$ & $\mathfrak{R}_{G^{N S}}^{2, Q}$ & $\mathfrak{R}_{G}^{2, \varrho}$ \\
\hline$\left(2^{-4}, 85\right)$ & 4.659 & 4.9459 \\
$\left(2^{-5}, 85\right)$ & & 7.3742 \\
\hline$\left(2^{-4}, 100\right)$ & 3.5376 & \\
\hline$\left(2^{-5}, 100\right)$ & & 5.8793 \\
\hline$\left(2^{-4}, 120\right)$ & 3.7551 & \\
$\left(2^{-5}, 120\right)$ & & \\
\hline$\left(2^{-5}, 130\right)$ & 4.0756 & 4.6976 \\
$\left(2^{-6}, 130\right)$ & & \\
\hline$\left(2^{-5}, 140\right)$ & 4.3845 & \\
$\left(2^{-6}, 140\right)$ & & \\
\hline
\end{tabular}

\section{Numerical Results}

The computational algorithm in Section 4 is applied and the implementation of the block-grid method is carried out using double precision. Let $\varepsilon_{h}=U_{h}^{k(M)}-u, \varepsilon_{h}^{(1)}=r^{1 / 2}\left(\left(\partial U_{h}^{k(M)} / \partial x\right)-\right.$ $(\partial u / \partial x)), \varepsilon_{h}^{(2)}=r^{3 / 2}\left(\left(\partial^{2} U_{h}^{k(M)} / \partial x^{2}\right)-\left(\partial^{2} u / \partial x^{2}\right)\right)$ be the errors in the "singular" part and $\varepsilon_{h}=u_{h}^{k(M)}-u$ be the error in the "nonsingular" part of the domain $G$.
TABLE 4: The order of convergence $R_{G^{N S}}^{6, \varrho}$, and $R_{G^{S}}^{6, \rho}$ when $h=2^{-\varrho}$.

\begin{tabular}{lcc}
\hline$\left(2^{-\varrho}, n\right)$ & $\mathfrak{R}_{G^{\mathrm{NS}}}^{6, Q}$ & $\mathfrak{R}_{G^{S}}^{6, Q}$ \\
\hline$\left(2^{-4}, 100\right)$ & 58.253 & 68.386 \\
$\left(2^{-5}, 100\right)$ & & \\
\hline$\left(2^{-4}, 145\right)$ & 58.788 & 97.005 \\
$\left(2^{-5}, 145\right)$ & & 65.711 \\
\hline$\left(2^{-5}, 125\right)$ & 61.164 & \\
$\left(2^{-6}, 125\right)$ & & \\
\hline$\left(2^{-5}, 130\right)$ & 64.140 & 88.926 \\
$\left(2^{-6}, 130\right)$ & & \\
\hline$\left(2^{-5}, 145\right)$ & 63.241 & \\
$\left(2^{-6}, 145\right)$ & & \\
\hline
\end{tabular}

In Table 1 the errors are given by the BGM when 5-point scheme with $S^{2}$ interpolation is used, and the iterations are terminated by using $\epsilon=5 \times 10^{-8}$. Table 2 represents the errors by the BGM when 9-point scheme is used with $S^{6}$ and the stopping criteria for the Schwarz's iterations is taken as $\epsilon=$ $5 \times 10^{-14}$.

The order of convergence in the "nonsingular" part, and the order of convergence in the "singular" part of $G$ are

$$
\begin{aligned}
& \mathfrak{R}_{G^{\mathrm{NS}}}^{k, \varrho}=\frac{\max _{G^{\mathrm{NS}}}\left|u_{2^{-\varrho}}^{k(M)}-u\right|}{\max _{G^{\mathrm{NS}}}\left|u_{2^{-(\varrho+1)}}^{k(M)}-u\right|}, \\
& \mathfrak{R}_{G^{\mathrm{S}}}^{k, \varrho}=\frac{\max _{G^{\mathrm{S}}}\left|U_{2^{-\varrho}}^{k(M)}-u\right|}{\max _{G^{\mathrm{S}}}\left|U_{2^{-(\varrho+1)}}^{k(M)}-u\right|},
\end{aligned}
$$

respectively, where $\varrho$ is a positive integer, $M$ is the final iteration number (Section 4), $k=2,6$. Taking $h=2^{-\varrho}$, $\varrho=4,5,6$, Tables 3 and 4 represent the order of convergence of the BGM in the "nonsingular" part and the "singular" part of the domain $G$ for $k=2$ and $k=6$, respectively.

The obtained numerical results in Tables 3 and 4 show that the order of convergence of the approximate solution is $\mathrm{O}\left(h^{2}\right)$ for the 5-point scheme with $S^{2}$ interpolation $(k=2)$ and it is $O\left(h^{6}\right)$ for the 9-point scheme with $S^{6}$ interpolation $(k=6)$ in the "nonsingular" part. In both tables, the order of convergence in the "singular" part is higher than the order of convergence in the "nonsingular" part of the domain, which 


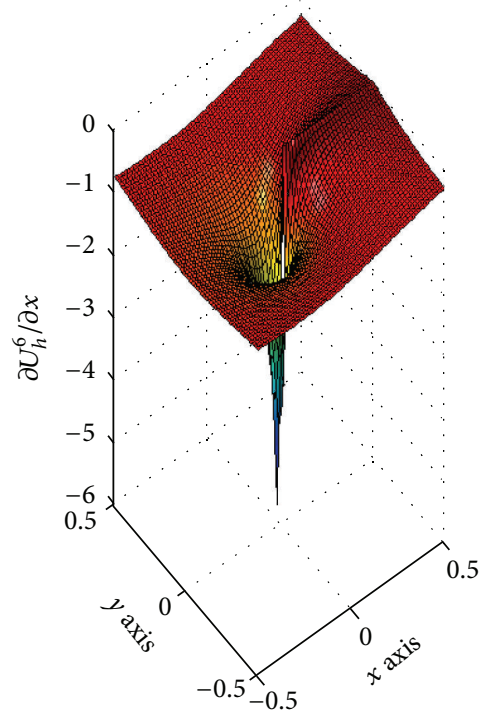

(a)

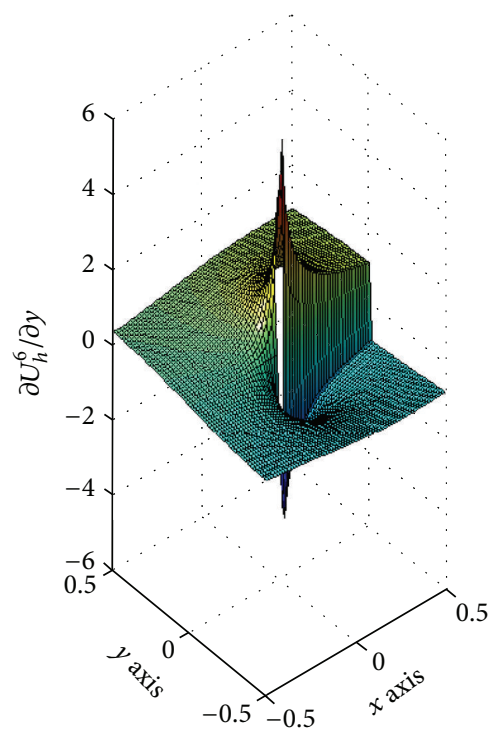

(b)

Figure 6: The first partial derivatives $\partial U_{h}^{6(M)} / \partial x$ and $\partial U_{h}^{6(M)} / \partial y$ in the "singular" part for $h^{-1}=64, n=140$.

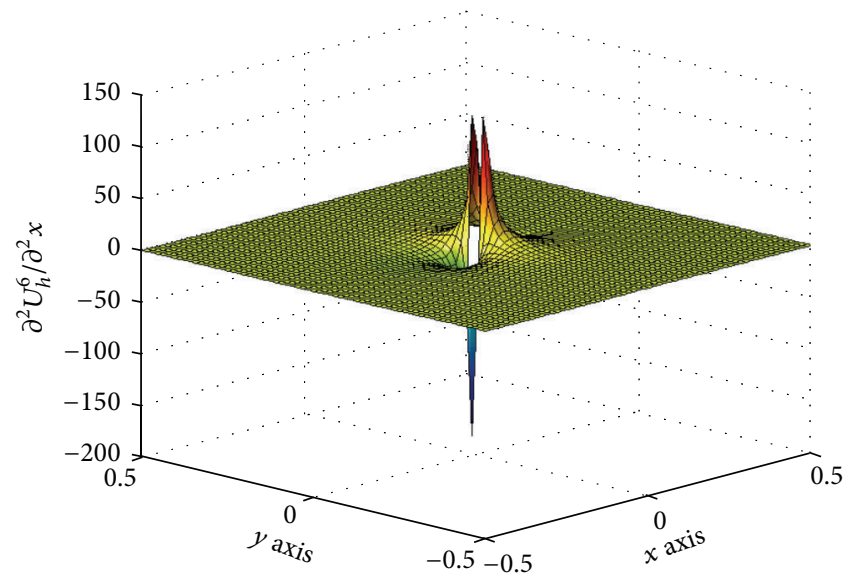

Figure 7: The second partial derivative $\partial^{2} U_{h}^{6(M)} / \partial x^{2}$ in the "singular" part for $h^{-1}=64, n=140$.

justifies the estimation (19) in Theorem 4. The errors with respect to the number of quadrature nodes $n$ in the "singular" part and in the "nonsingular" part by BGM for $k=2$, and $k=6$ are given in Figures 2 and 3, respectively. These figures demonstrate that the error in the "singular" part is less than the error in the "nonsingular" part for sufficiently large $n$ as it follows from the estimation (19) in Theorem 4. The graphical results in Figures 4-9 are obtained by the BGM when 9-point scheme is used with $S^{6}$ interpolation for $h^{-1}=64, n=140$. In Figure 4, the highly accurate approximate solution $U_{h}^{6(M)}$ and the exact solution $u$ is illustrated. Figure 5 represents the decrease of the error function $\left|U_{h}^{6(M)}-u\right|$ in the "singular" part of the domain as $r$ approaches to zero, which agrees with

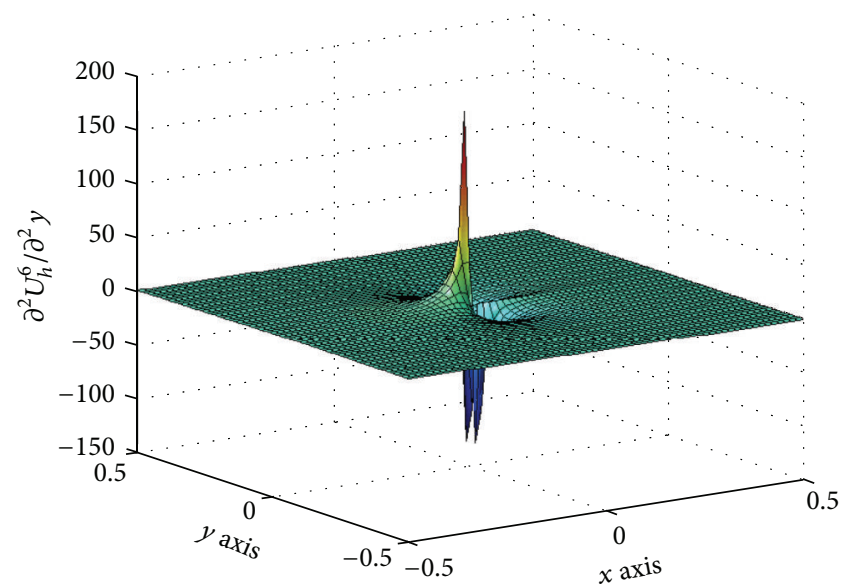

FIGURE 8: The second partial derivative $\partial^{2} U_{h}^{6(M)} / \partial y^{2}$ in the "singular" part for $h^{-1}=64, n=140$.

the estimation (19) in Theorem 4. Moreover, on the "singular" part, up to second order derivatives of the solution at grid points are approximated effectively by a simple differentiation of the function (31). The shapes of the first partial derivatives $\partial U_{h}^{6(M)} / \partial x, \partial U_{h}^{6(M)} / \partial y$ are demonstrated in Figure 6 and the shapes $\left(\partial^{2} U_{h}^{6(M)} / \partial x^{2}\right)\left(\partial^{2} U_{h}^{6(M)} / \partial y^{2}\right),\left(\partial^{2} U_{h}^{6(M)} / \partial x \partial y\right)$ are given in Figures 7, 8, and 9, respectively, to show the singular behaviour of the solution around the singular point.

5.1. Stress Intensity Factor. In engineering problems a very important constant is the so-called stress intensity factor $\sigma$. This constant gives a measure of "the amount of torsion the beam can endure before fracture occurs" $[10,13]$. On the basis 


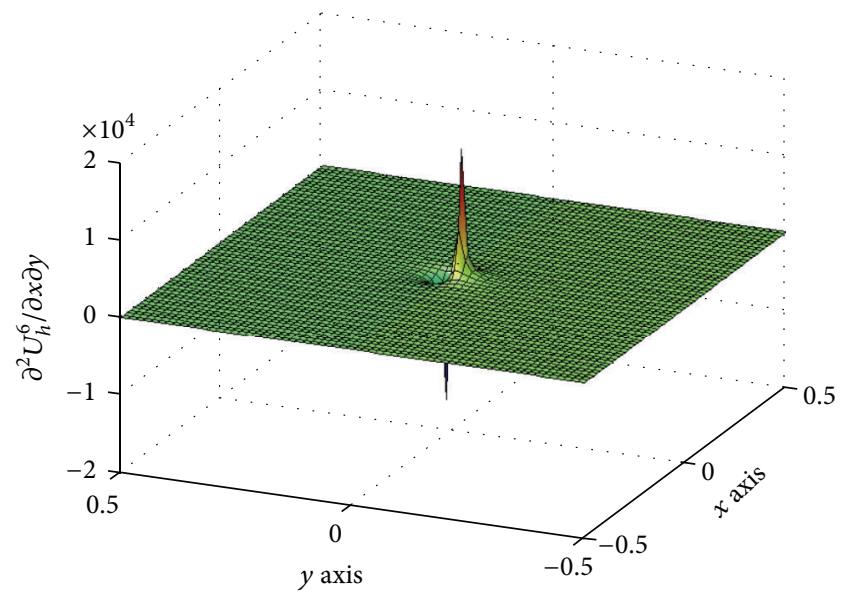

FIgURE 9: The mixed partial derivative $\partial^{2} U_{h}^{6(M)} / \partial x \partial y$ in the "singular" part for $h^{-1}=64, n=140$.

TABLE 5: The error of the stress intensity factor for fixed $n=140$.

\begin{tabular}{lcc}
\hline$h^{-1}$ & $\left|\sigma_{2}-\sigma\right|$ & $\left|\sigma_{6}-\sigma\right|$ \\
\hline 16 & $3.4791 \times 10^{-6}$ & $3.7655 \times 10^{-10}$ \\
\hline 32 & $3.0364 \times 10^{-6}$ & $1.9451 \times 10^{-12}$ \\
64 & $1.4899 \times 10^{-8}$ & $5.9952 \times 10^{-15}$ \\
\hline
\end{tabular}

of (31) we give a simple and highly accurate formula for the stress intensity factor $\sigma$ denoting by $\sigma_{k}$ for $k=2,6$ :

$$
\begin{aligned}
\sigma_{k} & =\lim _{r \rightarrow 0} \frac{U_{h}^{k(M)}(r, \pi)}{r^{1 / 2}} \\
& =\frac{2}{n \sqrt{(0.93)}} \sum_{q=1}^{n} u_{h}^{k(M)}\left(0.93, \theta^{q}\right) \sin \frac{\theta^{q}}{2},
\end{aligned}
$$

where $\theta^{q}=(q-1 / 2) 2 \pi / n$ and $M$ is the final iteration number. The exact value of the stress intensity factor $\sigma$ is $\sqrt{2}$. For fixed number of quadrature nodes $n=140$, the second column in Table 5 represents the error of the stress intensity factor when 5-point scheme is used with $S^{2}$ and the last column represents this error when 9-point scheme is used with $S^{6}$.

\section{Conclusion}

For the solution of the Laplace equation on polygons with a slit, the real characteristics of the block-grid method is investigated. The given polygon is decomposed into five overlapping rectangles and one sector. In the sector, we approximate the special integral representation of the solution, which takes into account the behaviour of the exact solution near the end point of the slit. On the rectangles, to approximate Laplace's equation on square grids either 5-point scheme is used which is simpler by means of sparsity, or 9-point scheme is used, which gives a highly accurate approximation. In correspondence with the finite difference scheme used, a gluing together of the subsystems is carried out effectively by a sufficiently simple linear interpolation $S^{2}$, or a highly accurate interpolation $S^{6}$. By choosing the step size $h=2^{-4}, 2^{-5}, 2^{-6}$, the obtained numerical results show that the order of convergence of the approximate solution is $O\left(h^{2}\right)$ for the 5-point scheme with $S^{2}$ and it is $O\left(h^{6}\right)$ for the 9-point scheme with $S^{6}$ in the "nonsingular" part. The results also show that the order of convergence in the "singular" part is higher than the order of convergence in the "nonsingular" part of the domain. This conclusion justifies the theoretical results obtained in [2-5]. Moreover, the shapes up to the second-order derivatives of the highly accurate solution obtained by the BGM are shown to display the singular behaviour at the end point of the slit. Finally the stress intensity factor is approximated by the given highly accurate formula.

\section{References}

[1] Z. C. Li, Combined Methods for Elliptic Problems with Singularities, Interfaces and Infinities, Kluwer Academic, London, UK, 1998.

[2] A. A. Dosiyev, "A block-grid method for increasing accuracy in the solution of the Laplace equation on polygons," Russian Academy of Sciences, vol. 45, no. 2, pp. 396-399, 1992.

[3] A. A. Dosiyev, "A block-grid method of increased accuracy for solving Dirichlet's problem for Laplace's equation on polygons," Computational Mathematics and Mathematical Physics, vol. 34, no. 5, pp. 591-604, 1994.

[4] A. A. Dosiyev, "The high accurate block-grid method for solving Laplace's boundary value problem with singularities," SIAM Journal on Numerical Analysis, vol. 42, no. 1, pp. 153-178, 2004.

[5] A. A. Dosiyev and S. Cival, "A difference-analytical method for solving Laplace's boundary value problem with singularities," in Proceedings of Conference on Applications of Dynamical Systems, pp. 339-360, Antalya, Turkey, July 2004.

[6] A. A. Dosiyev and S. C. Buranay, "A fourth order accurate difference-analytical method for solving Laplace's boundary value problem with singularities," in Mathematical Methods in Engineers, K. Tas, J. A. T. Machado, and D. Baleanu, Eds., pp. 167-176, Springer, Berlin, Germany, 2007.

[7] E. A. Volkov, "An exponentially converging method for solving Laplace's equation on polygons," Mathematics of the USSRSbornik, vol. 37, no. 3, pp. 295-325, 1980.

[8] E. A. Volkov, Block Method for Solving the Laplace Equation and Constructing Conformal Mappings, CRC Press, Boca Raton, Fla, USA, 1994.

[9] N. M. Wigley, "An efficient method for subtracting off singularities at corners for Laplace's equation," Journal of Computational Physics, vol. 78, no. 2, pp. 369-377, 1988.

[10] G. J. Fix, S. Gulati, and G. I. Wakoff, "On the use of singular functions with finite element approximations," Journal of Computational Physics, vol. 13, pp. 209-228, 1973.

[11] W. Wasow, "On the truncation error in the solution of Laplace's equation by finite differences," Journal of Research of the National Bureau of Standards, vol. 48, pp. 345-348, 1952.

[12] S. E. Romanova, "An efficient method for the approximate solution of the Laplace difference equation on rectangular domains," Zhurnal Vychislitel'noi Matematiki i Matematicheskoi Fiziki, vol. 23, no. 3, pp. 660-673, 1983.

[13] A. A. Dosiyev and S. C. Buranay, "On solving the crackedbeam problem by block method," Communications in Numerical Methods in Engineering with Biomedical Applications, vol. 24, no. 11, pp. 1277-1289, 2008. 


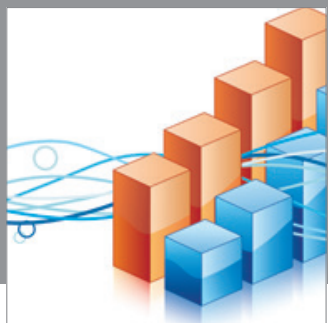

Advances in

Operations Research

mansans

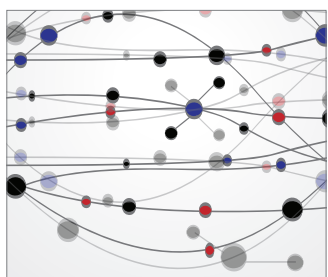

The Scientific World Journal
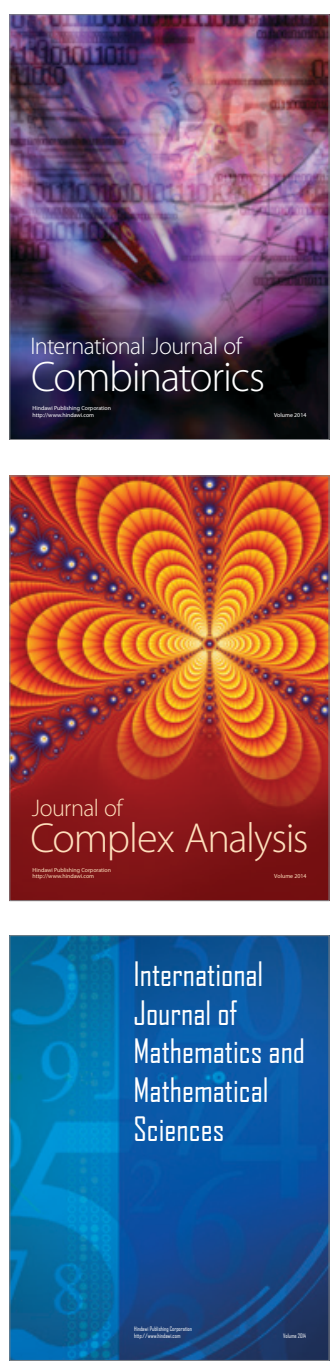
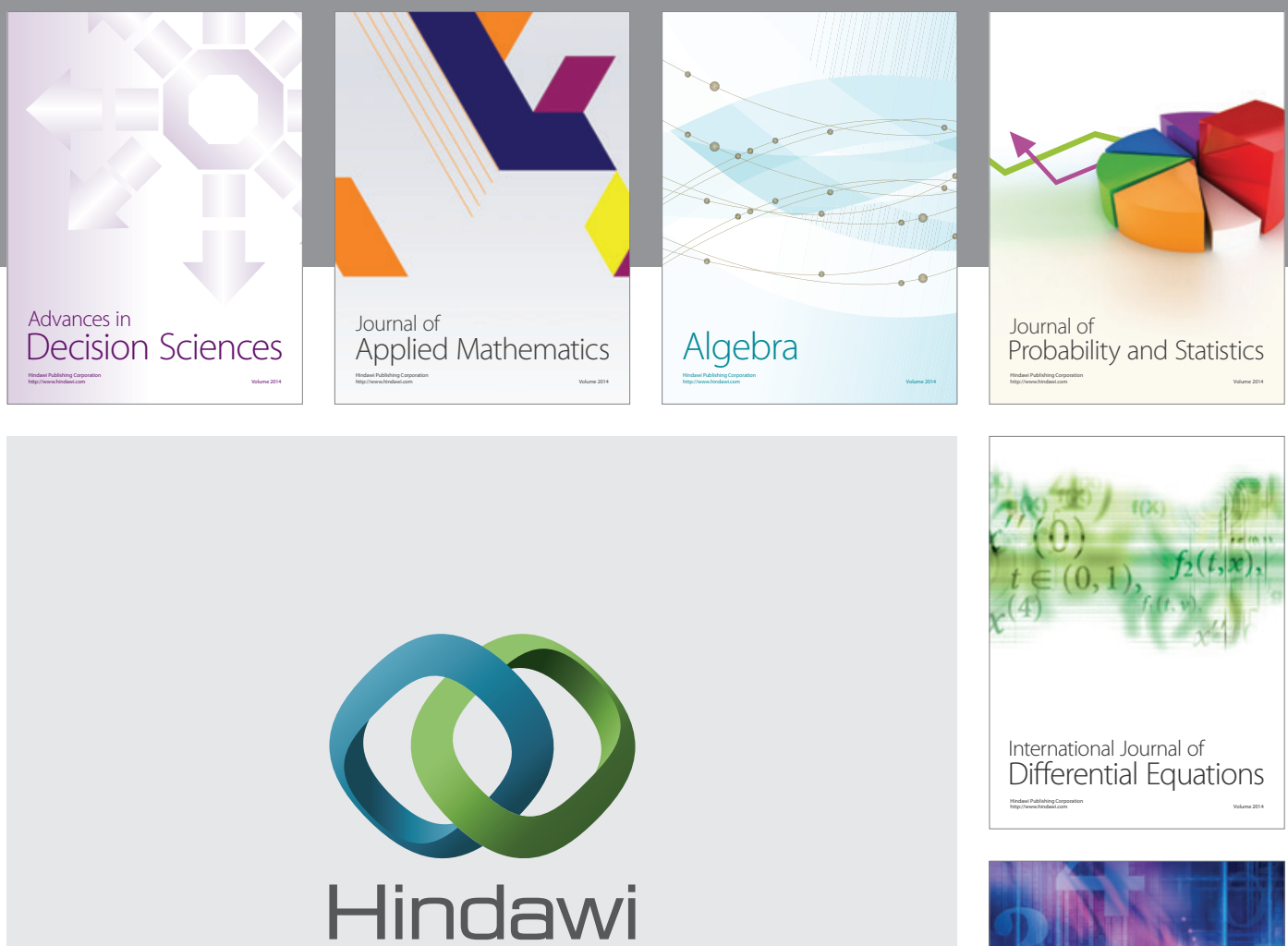

Submit your manuscripts at http://www.hindawi.com
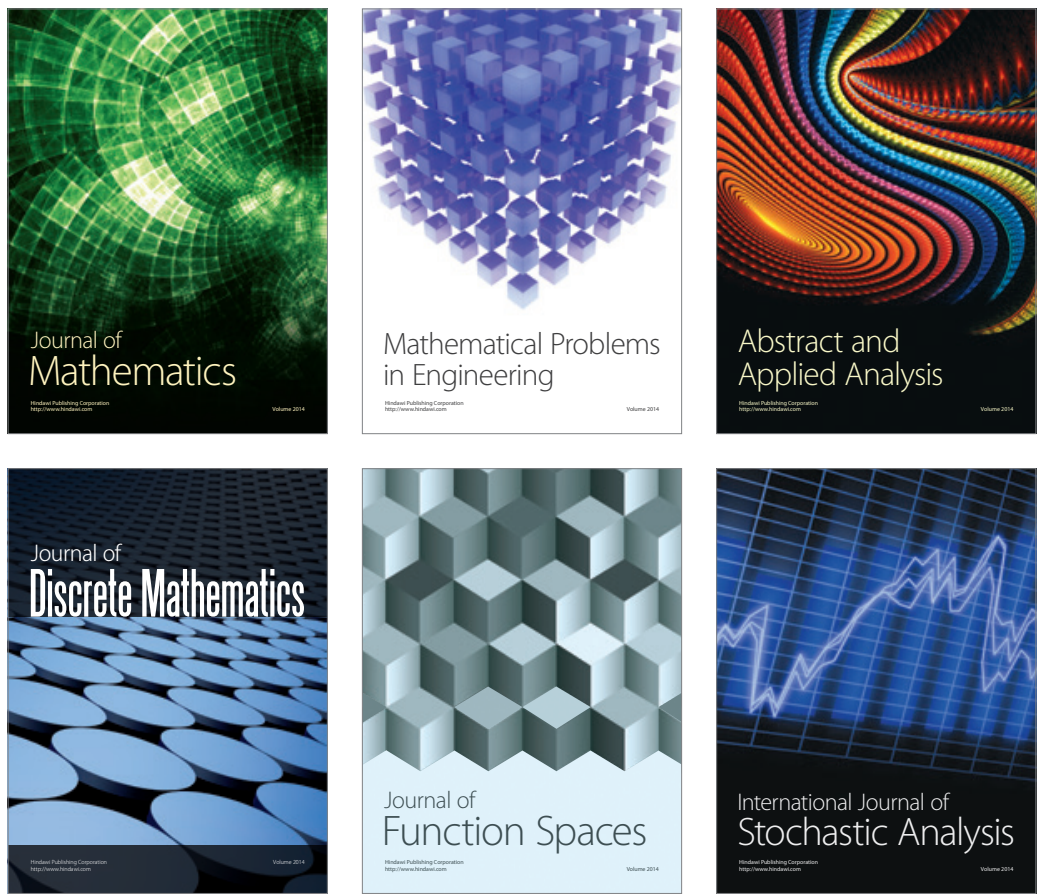

Journal of

Function Spaces

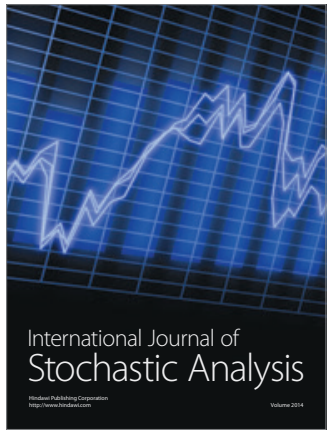

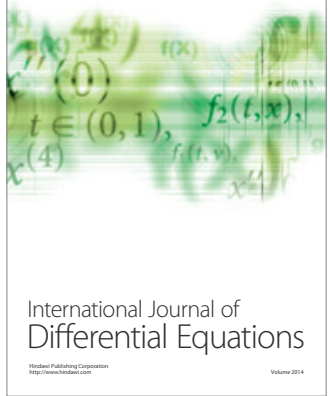
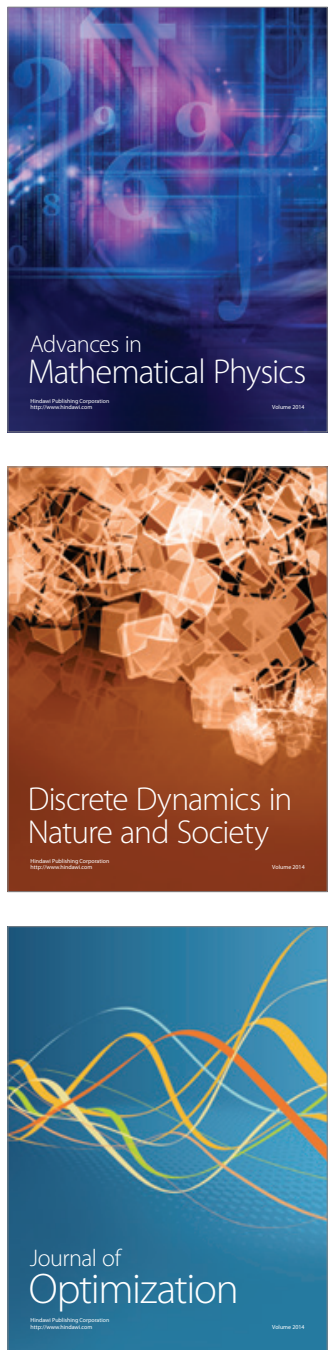